\title{
Effects of pulsing of the target tone on the audibility of partials in inharmonic complex
} tones

\author{
Moore, Brian C.J.; Glasberg, Brian R.; Jepsen, Morten Løve
}

Published in:

Journal of the Acoustical Society of America

Link to article, DOI:

10.1121/1.3109997

Publication date:

2009

Document Version

Publisher's PDF, also known as Version of record

Link back to DTU Orbit

Citation $(A P A)$ :

Moore, B. C. J., Glasberg, B. R., \& Jepsen, M. L. (2009). Effects of pulsing of the target tone on the audibility of partials in inharmonic complex tones. Journal of the Acoustical Society of America, 125(5), 3194-3204.

https://doi.org/10.1121/1.3109997

\section{General rights}

Copyright and moral rights for the publications made accessible in the public portal are retained by the authors and/or other copyright owners and it is a condition of accessing publications that users recognise and abide by the legal requirements associated with these rights.

- Users may download and print one copy of any publication from the public portal for the purpose of private study or research.

- You may not further distribute the material or use it for any profit-making activity or commercial gain

- You may freely distribute the URL identifying the publication in the public portal 


\title{
Effects of pulsing of the target tone on the audibility of partials in inharmonic complex tones
}

\author{
Brian C. J. Moore ${ }^{\text {a) }}$ and Brian R. Glasberg \\ Department of Experimental Psychology, University of Cambridge, Downing Street, Cambridge CB2 3EB, \\ England \\ Morten L. Jepsen \\ Centre for Applied Hearing Research, Department of Electrical Engineering, Technical University of \\ Denmark, DK-2800 Kgs. Lyngby, Denmark
}

(Received 27 June 2008; revised 9 March 2009; accepted 10 March 2009)

\begin{abstract}
The audibility of partials was measured for complex tones with partials uniformly spaced on an $\mathrm{ERB}_{\mathrm{N}}$-number scale. On each trial, subjects heard a sinusoidal "probe" followed by a complex tone. The probe was mistuned downwards or upwards (at random) by 3\% or $4.5 \%$ from the frequency of one randomly selected partial in the complex (the "target"). The subject indicated whether the target was higher or lower in frequency than the probe. The probe and the target were pulsed on and off and the ramp times and inter-pulse intervals were systematically varied. Performance was better for longer ramp times and longer inter-pulse intervals. In a second experiment, the ability to detect which of two complex tones contained a pulsed partial was measured. The pattern of results was similar to that for experiment 1 . A model of auditory processing including an adaptation stage was able to account for the general pattern of the results of experiment 2 . The results suggest that the improvement in ability to hear out a partial in a complex tone produced by pulsing that partial is partly mediated by a release from adaptation produced by the pulsing, and does not result solely from reduction of perceptual confusion.
\end{abstract}

(C) 2009 Acoustical Society of America. [DOI: 10.1121/1.3109997]

PACS number(s): 43.66.Fe, 43.66.Hg, 43.66.Mk [RLF] Pages: 3194-3204

\section{INTRODUCTION}

If attention is directed in an appropriate way, human listeners have some ability to "hear out" individual partials in complex tones (Helmholtz, 1954). Partials that can be heard out in this way are referred to as "resolved." It is often assumed that the ability to hear out partials depends at least partly on the sharpness of the auditory filters (Plomp, 1964; Plomp and Mimpen, 1968; Moore and Ohgushi, 1993; Moore et al., 2006c), although factors such as the musical experience of the listeners (Soderquist, 1970; Fine and Moore, 1993) and phase locking (Moore and Ohgushi, 1993; Hartmann et al., 1990; Hartmann and Doty, 1996; Moore et al., 2006c) may also play a role. It has been proposed (Moore and Ohgushi, 1993; Moore, 2003) that, for a complex tone with equal-amplitude partials, each "inner" partial (i.e., excluding the highest and lowest partials) can be heard out with $75 \%$ accuracy when it is separated from neighboring partials by about $1.25 \mathrm{ERB}_{\mathrm{N}}$, where $\mathrm{ERB}_{\mathrm{N}}$ refers to the equivalent rectangular bandwidth of the auditory filter as determined for young normally hearing listeners at moderate sound levels (Glasberg and Moore, 1990; Moore, 2003). This is consistent with the finding of several studies that, for harmonic complex tones with fundamental frequencies in the

\footnotetext{
a) Author to whom correspondence should be addressed. Electronic mail: bcjm@cam.ac.uk
}

range 100-400 Hz, harmonics with numbers up to about 5-8 can be heard out (Plomp, 1964; Plomp and Mimpen, 1968; Fine and Moore, 1993).

Recently, Bernstein and Oxenham (2003, 2006, 2008) suggested that the ability to hear out harmonics extends to harmonics with numbers up to about 10 . They used a twoalternative forced-choice task similar to that employed by Roberts and Bregman (1991) and Moore and Ohgushi (1993). On each trial, subjects had to indicate whether a sinusoidal "probe" was higher or lower in frequency than the nearest harmonic in the complex tone that was presented after the probe; this is referred to as the "target" harmonic. Bernstein and Oxenham $(2003,2006,2008)$ suggested that the results of previous studies using this or similar methods might have been influenced by cognitive or attentional factors. For example, subjects may have difficulty in deciding which harmonic in the complex to compare with the probe. Subjects may also have difficulty in overcoming perceptual fusion of the harmonics in the complex caused by their harmonicity and by their synchronous gating (Moore et al., 1986). For brevity, we will refer to all such effects as "confusion" effects. Musically trained listeners may be less susceptible to such confusion effects, which could explain why they perform better than non-musicians in tasks requiring them to hear out partials (Soderquist, 1970; Fine and Moore, 1993). To overcome these difficulties, in the experiment of Bernstein and Oxenham (2003) both the probe and the target harmonic were pulsed on and off. This was done "to help overcome any nonperipheral limitations and to encourage 
perceptual segregation, while not affecting peripheral resolvability" (p. 3325). Performance on this task was very good $(>90 \%)$ for the lower harmonics, but decreased with increasing harmonic number, reaching $75 \%$ correct for about the tenth harmonic, for fundamental frequencies of 100 and $200 \mathrm{~Hz}$. Thus, the upper limit found by Bernstein and Oxenham (2003) was markedly higher than found in earlier studies, in which the target harmonic was not pulsed.

Bernstein and Oxenham (2003) assumed that the pulsing would not affect peripheral factors. However, it is not clear that this assumption is valid (Hartmann and Goupell, 2006; Moore et al., 2006c). When a tone is presented continuously, the response of single neurons in the auditory nerve to that tone declines over time, an effect called adaptation (Smith, 1977). For a continuous harmonic complex tone, the responses to all harmonics would show adaptation. However, when a tone is turned off and then back on again, the firing rate of single neurons often shows an initial peak when the tone is turned back on, presumably as a consequence of recovery from adaptation (Smith, 1977). Similarly, if one partial in a complex tone is turned on after the remaining partials, the discharge rate of neurons tuned to the frequency of the delayed-onset partial shows a distinct increase relative to the rate obtained when all partials are turned on at the same time (Palmer et al., 1995). Palmer et al. (1995, p. 1787) concluded that "the action of adaptation of the discharge of auditory-nerve fibers can increase the spectral contrast of an introduced component." It is possible therefore that the ability of subjects to hear out the target harmonic in the experiments of Bernstein and Oxenham (2003, 2006, 2008) was enhanced by a recovery from adaptation produced by pulsing the target harmonic on and off.

Hartmann and Goupell (2006) studied the ability of subjects to make pitch matches to a pulsed harmonic in a complex tone. They found that the highest harmonic for which a match could be made reliably depended on the relative phases of the harmonics. When subjects were allowed to listen to harmonic complexes with different starting phases of the harmonics, they were able to select a "favorable" set of phases that allowed a selected (pulsed) harmonic to be heard out more easily and matched more accurately than for other phase selections. For favorable phase selections, subjects could make reliable pitch matches for harmonics up to the 20th. Hartmann and Goupell (2006) argued that harmonics above the tenth would not be spectrally resolved, and that the pulsing enabled pitch matching to harmonics that would not normally be resolved.

In the present experiment, we assessed the possible role of recovery from adaptation by using a similar task to that of Bernstein and Oxenham (2003, 2006, 2008), but exploring the effect of varying the interval between pulses and the rise/fall time of the pulses. If the pulsing produces a release from adaptation that makes the task easier, then shortening the interval between pulses or decreasing the rise/fall time of the pulses should lead to a worsening in performance, because both of these manipulations would lead to a reduced release from adaptation (Smith, 1977; Smith, 1979; Westerman and Smith, 1984).
If pulsing the target mainly influences confusion effects, for example, by helping the subject to determine which harmonic to attend to, then changing the interval between pulses or changing the rise/fall time of the pulses might also have some influence on the results, by making the pulsed component more or less salient. Bernstein and Oxenham (2003) did not discuss this possibility, implicitly assuming that the interval between pulses and the rise/fall time of the pulses used by them were sufficient to remove any effects of confusion. If resolution of confusion is the sole effect of pulsing the target, then one would expect performance to reach a plateau if the inter-pulse interval is sufficiently long. For closely spaced partials, for which peripheral resolvability should limit performance, this plateau should occur for a performance level well below $100 \%$. On the other hand, if part of the effect of pulsing of the target is to produce a release from adaptation, one might expect to see a progressive improvement in performance with increasing inter-pulse interval, even when the partials are closely spaced. To test which of these predictions was closer to the truth, we included interpulse intervals longer than those employed by Bernstein and Oxenham (2003, 2006, 2008).

As a further way of assessing the relative importance of peripheral adaptation as opposed to central confusion, we used two approaches. First, we conducted a second experiment, in which we measured the ability to detect which of two complex tones contained a pulsed partial, varying the interval between pulses and changing the rise/fall time of the pulses in the same way as for experiment 1 . We argued that central factors should play a much smaller role in this case, as the stimuli were chosen so that there was minimal uncertainty about which component in the complex tone to attend to. If the pattern of results was similar for the two experiments, this would support the idea that peripheral factors such as release from adaptation were the main cause of the effects of varying inter-pulse interval and rise/fall time. As a further test of this idea, we used the stimuli as input to a model of auditory processing that includes an adaptation stage (Dau et al., 1996a), to explore whether the pattern of results could be predicted by the internal representation (IR) produced by the model.

In contrast to Bernstein and Oxenham (2003) and Hartmann and Goupell (2006), who used harmonic complex tones, we used complex tones with partials uniformly spaced on an $\mathrm{ERB}_{\mathrm{N}}$-number scale (see below for details), which were therefore inharmonic. This was done for three reasons. First, it reduced the tendency for the components to fuse based on their harmonicity (Moore et al., 1986). Second, it avoided the possibility that subjects might infer which harmonic in a complex tone was closest in frequency to the probe without actually hearing out the target partial, based on a conscious or implicit knowledge of the frequencies of tones that form a harmonic series. Finally, the waveform of the inharmonic tones was not periodic, so it was unlikely that the results would be influenced by the specific set of random starting phases chosen for the partials. 
TABLE I. Frequencies $(\mathrm{Hz})$ of the partials in the complex tones for each spacing used. The frequency of the middle partial is given in bold type.

\begin{tabular}{|c|c|c|c|c|c|c|c|c|c|c|c|}
\hline \multirow[b]{2}{*}{ Spacing } & \multicolumn{11}{|c|}{ Partial number } \\
\hline & 1 & 2 & 3 & 4 & 5 & 6 & 7 & 8 & 9 & 10 & 11 \\
\hline $1.5 E$ & 408 & 520 & 652 & 806 & 988 & 1201 & 1452 & 1747 & 2094 & 2501 & 2980 \\
\hline $1.0 E$ & 605 & 700 & 806 & 924 & 1055 & 1201 & 1364 & 1545 & 1747 & 1972 & 2222 \\
\hline $0.75 E$ & 726 & 806 & 893 & 988 & 1090 & 1201 & 1322 & 1452 & 1594 & 1747 & 1913 \\
\hline
\end{tabular}

\section{EXPERIMENT 1: EFFECT OF PULSING ON THE ABILITY TO HEAR OUT PARTIALS}

\section{A. General method}

The method was similar to that used by Roberts and Bregman (1991), Moore and Ohgushi (1993), Bernstein and Oxenham (2003), and Moore et al. (2006c). On each trial subjects were presented with a sinusoidal tone followed by a complex tone. The sinusoid will be referred to as the probe. Subjects were told that the probe was close in frequency to one of the partials in the complex tone (the target), but was actually slightly higher or lower in frequency than the target. On half the trials, chosen at random, the probe was higher in frequency than the target by $\Delta f$, and on the other half it was lower by $\Delta f$. Subjects were asked to indicate, by pressing the appropriate button on the response box, whether the "closest" partial in the complex was higher or lower in frequency than the probe. Correct-answer feedback was provided after each trial by lights on the response box. The partial that was "probed" was varied randomly from trial to trial. The frequencies of all partials in the complex tone were randomly varied (roved) from trial to trial by multiplying them by a factor randomly chosen within the range 0.9-1.1, while keeping the frequency ratios between partials fixed. The frequency of the probe was multiplied by the same factor.

Before testing started, subjects were given training, starting with easy conditions and working toward more difficult conditions. Training started with a "complex" tone containing a single sinusoid with a nominal frequency of 1000 Hz. In this case, the task was a simple frequency discrimination task (but with roving, as described above). Then, the number of partials in the complex tone was increased to 2, with widely separated frequencies. When subjects scored better than $90 \%$ with this complex, the number of partials was increased to 3 with widely spaced frequencies, and then to 5. Some subjects who found the task to be easy skipped the training with intermediate numbers of partials. Subjects were then given training runs with the complex tones to be used in the main experiment that contained 11 partials. After this training, performance appeared to remain largely stable.

In the main experiment, each partial in a complex was probed ten times in a given run, five times with the probe lower in frequency than the relevant partial in the complex, and five times with it higher. Five runs were obtained for each complex tone, giving a total of 50 judgments for each partial. In a few cases, when scores for the first few runs were close to $100 \%$ for all partials, testing stopped after fewer than five runs.

\section{B. Stimuli}

The partials in each complex tone were equally spaced on an $\mathrm{ERB}_{\mathrm{N}}$-number scale. The relationship between ERB $_{\mathrm{N}}$-number, $E$, and frequency, $f(\mathrm{~Hz})$, was assumed to be as suggested by Glasberg and Moore (1990):

$$
E=21.4 \log _{10}(0.00437 f+1) .
$$

The spacings used were $0.75 E, 1.0 E$, and $1.5 E$. The mean frequency of the central partial was always $1201 \mathrm{~Hz}$, corresponding to $E=17$. The mean frequencies of all partials for each spacing used are given in Table I. All of the complex tones contained 11 partials. The values of $\Delta f$ were $3 \%$ of the frequency of the target partial for the spacing of $0.75 \mathrm{E}$ and $4.5 \%$ of the frequency of the target partial for the other spacings. The value of $\Delta f$ was made smaller for the spacing of $0.75 E$ to ensure that the probe tone was always closer in frequency to the target partial than to any other partial. For example, when the ninth partial $(1594 \mathrm{~Hz})$ in the complex tone was the target, a value of $\Delta f$ of $+3 \%$ led to the probe being 3\% higher in frequency than the target partial and $6 \%$ lower than the tenth partial $(1747 \mathrm{~Hz})$.

The probe tone and all partials in the complex tone each had an overall duration of $1000 \mathrm{~ms}$, with 20-ms raised-cosine rise/fall ramps. The inter-stimulus interval was $300 \mathrm{~ms}$. The non-target partials in the complex were always uninterrupted for the 1000-ms duration. In condition 1, the probe tone and the target partial were also uninterrupted for the 1000-ms duration. This condition is very similar to that used previously by Moore et al. (2006c). In the remaining conditions, both the probe tone and the target partial were pulsed off and on, with the same off-on pattern for the probe and target. The following conditions were used, all with raised-cosine ramps (note that these ramp times refer to the ramps within the pulsing pattern; the very first and very last ramps always lasted $20 \mathrm{~ms})$ :

(2) 10-ms ramps, 0-ms off time, 306.7-ms steady state pulse duration,

(3) 20-ms ramps, 0-ms off time, 293.3-ms steady state pulse duration,

(4) 40-ms ramps, 0-ms off time, 266.7-ms steady state pulse duration,

(5) 20-ms ramps, 50-ms off time, 260-ms steady state pulse duration [as used by Bernstein and Oxenham (2003)], and

(6) 20-ms ramps, 100-ms off time, 226.7-ms steady state pulse duration.

The pulsing of the probe was always relatively easy to 


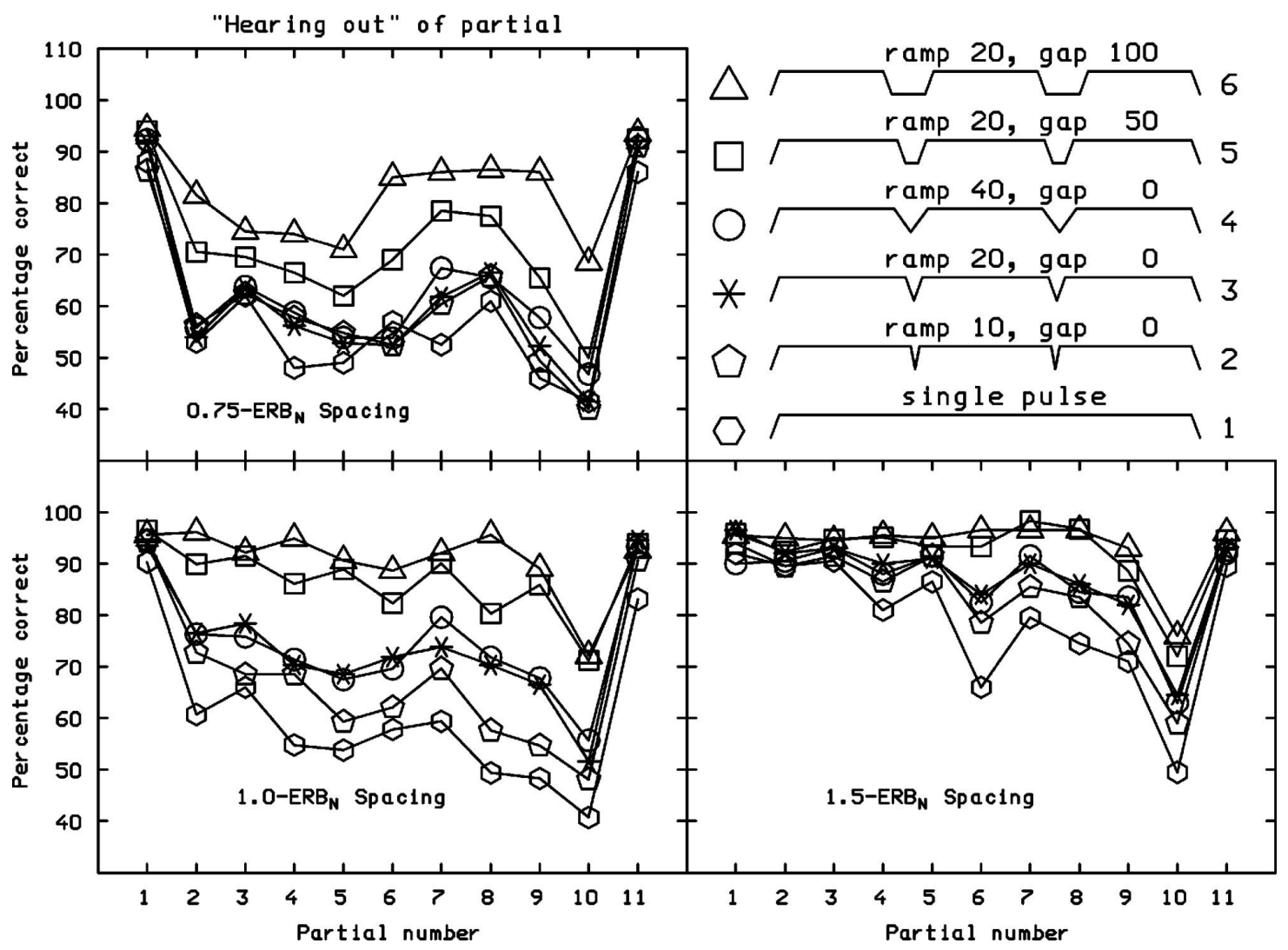

FIG. 1. Scores averaged across subjects for experiment 1. The percentage correct is plotted as a function of partial number (see Table I for frequencies of partials). Within each panel, the parameter is the pulsing pattern of the probe tone and the target tone within the complex (illustrated schematically on the top-right). Each panel shows results for one spacing of the partials, as indicated in the key.

hear, even for condition 2, which used the shortest ramps and off time. The probe tone and each partial in the complex tone all had the same level, which was $50 \mathrm{~dB}$ sound pressure level (SPL). The starting phases of the partials in each complex tone were chosen randomly for each trial.

Stimuli were generated digitally on-line using a TuckerDavis Technologies (TDT) system II. The stimuli were played through a 16-bit digital-to-analog converter (TDT, DD1) at a $50-\mathrm{kHz}$ sampling rate, lowpass filtered at $8 \mathrm{kHz}$ (Kemo VBF8/04), attenuated (TDT, PA4), and presented via a headphone buffer (TDT, HB6), a manual attenuator (Hatfield 2125), and one earpiece of a Sennheiser HD580 headphone, which has a diffuse-field response. Levels specified are equivalent diffuse-field levels. Levels at the eardrum would have been higher for frequencies around $3000 \mathrm{~Hz}$ (Moore et al., 1998). Subjects were tested individually in a double-walled sound-attenuating chamber.

\section{Subjects}

Four subjects (one male, three female) were used, all with no reported history of hearing disorders. One was author BG. Their absolute thresholds were better than $20 \mathrm{~dB}$ $\mathrm{HL}$ for audiometric frequencies from 250 to $8000 \mathrm{~Hz}$ (ISO $389-8,2004)$. Their ages ranged from 21 to 62 years and all had some degree of musical training. Musically trained subjects were chosen, since subjects without such training often have difficulty with this task, especially when the probe and target tone are not pulsed (Fine and Moore, 1993). All subjects except author BG were paid for their participation.

\section{Results and discussion}

Although there were some individual differences, as have also been found in previous similar studies, the general pattern of results was similar across subjects. Mean results are shown in Fig. 1. The scores are plotted as a function of partial number and are averaged across the cases when the probe was lower in frequency than the target and when it was higher in frequency. Each panel shows results for one spacing. The different conditions of pulsing are indicated schematically at the right of the figure. The scores for the lowest and highest (edge) partials were high for all spacings and for all pulsing patterns. These high scores are consistent with other research showing that edge partials are easier to hear out from complex tones than inner partials (Plomp, 1964; Moore, 1973; Moore et al., 1984; Moore and Ohgushi, 1993; Moore et al., 2006c; Gockel et al., 2007).

Scores for the inner partials generally worsened when the partial number was above 8 , corresponding to a frequency of approximately 1500-1700 Hz. A similar trend was found by Moore and Ohgushi (1993) and by Moore et al. (2006c); the trend seems to be related to absolute frequency rather than to partial number. The trend for worse performance at higher frequencies is consistent with the finding of Plomp (1964) and of Plomp and Mimpen (1968) that the frequency spacing necessary for a given partial to be heard 
out was greater than a critical band at high frequencies but less than a critical band at low frequencies. This trend may indicate a role of phase locking in the ability to hear out partials, since phase locking becomes less precise for frequencies above about $1500 \mathrm{~Hz}$ (Johnson, 1980; Palmer and Russell, 1986).

The scores for the second-highest partial in the complex tone (partial 10) were especially low and for the two smaller spacings were below the chance level of 50\% for some conditions, indicating systematic errors. Such a pattern has been observed previously (Moore et al., 2006c). These low scores can be partly explained in terms of the high salience of the pitch of the highest partial. This high salience may be produced by phase locking to that partial in neurons tuned above the frequency of the partial (Moore, 2003). It appears that, when the frequency of the probe was above the frequency of the second-highest partial, the pitch of the probe was often judged relative to that of the highest partial rather than that of the closest partial (the target). Hence, subjects consistently and erroneously responded that the partial in the complex was higher in pitch. It is of interest that a dip in performance for the tenth partial occurred even for the pulsing pattern with the longest inter-pulse interval (condition 6). It appears that the extra salience of the target partial produced by pulsing it on and off was not sufficient to overcome the effect of the intrinsic high salience of the highest partial in the complex.

The results for individual subjects showed systematic irregularities in the pattern of results as a function of partial number. For example, for the $1-\mathrm{ERB}_{\mathrm{N}}$ spacing, one subject showed especially low scores for the sixth partial and another subject showed especially low scores for the fifth partial. Such irregularities have been observed previously, and it has been proposed that they result from irregularities in middle-ear transmission, which may make some partials easier to hear out than others (Moore and Ohgushi, 1993; Moore et al., 2006c).

Performance generally improved with increasing spacing, as expected. Importantly, performance was also strongly influenced by the pattern of pulsing of the target partial. Performance was worst overall when the target was presented as a single long pulse (hexagons) and was best overall for the condition where the inter-pulse interval was $100 \mathrm{~ms}$ (triangles). For example, for the $1-\mathrm{ERB}_{\mathrm{N}}$ spacing, scores for partials $2-8$ were close to chance for the former and above $90 \%$ for the latter.

To illustrate the overall effect of spacing and of the pulsing pattern of the target tone, the data were averaged across all inner partials, except for the tenth (since the latter led to anomalous results, as discussed above). The outcome is shown in Fig. 2. For each pulsing pattern, the scores increased with increasing spacing, and for each spacing the scores were strongly influenced by the pulsing pattern.

To assess the statistical significance of the effects described above, the data were transformed to rationalized arcsine units (RAU; Studebaker, 1985) and then to a withinsubjects analysis of variance (ANOVA), with factors type of pulsing (six types), partial number (2-9), and spacing $(0.75 E, 1 E$, or $1.5 E)$. The main effect of type of pulsing was

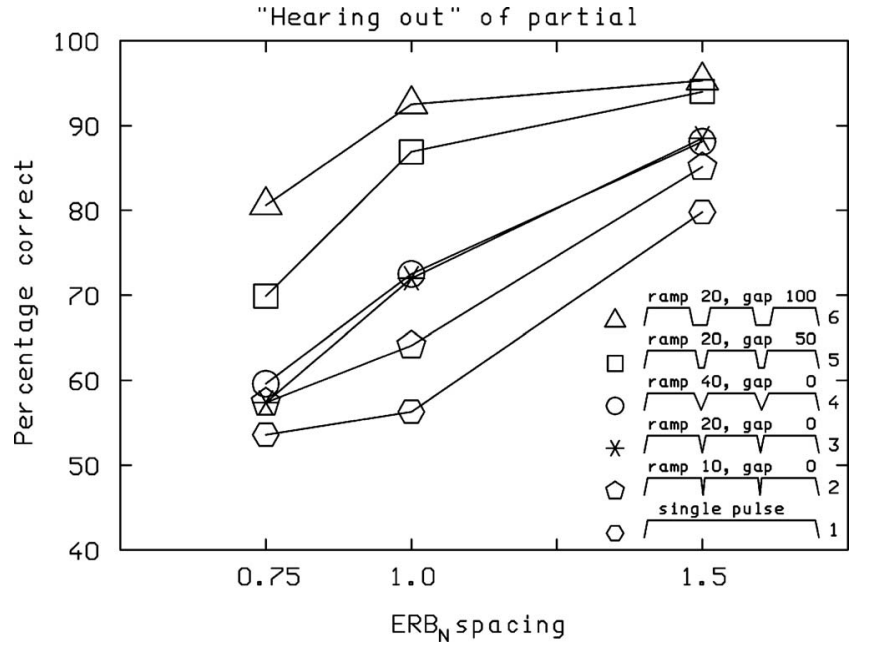

FIG. 2. Scores for experiment 1 averaged across all inner partials and plotted as a function of the spacing of the partials. The parameter is the pulsing pattern of the probe tone and the target tone within the complex, as indicated in the key.

significant: $F(5,15)=67.13, p<0.001$. The main effect of partial number was not significant: $F(7,21)=1.96, p=0.11$. The main effect of spacing was not significant: $F(2,6)$ $=4.04, p=0.077$. The interaction of type of pulsing and spacing was significant: $F(10,30)=2.30, p=0.038$, but accounted for only about $2 \%$ of the variance in the data. The interaction of partial number and pulsing pattern was also significant: $F(35,105)=1.89, p=0.007$, but again accounted for less than $2 \%$ of the variance in the data. No other interactions were significant.

The large effect of the pulse pattern of the probe and target tones indicates that the effect of pulsing is not simply to remove confusion effects in an all-or-none manner. If the effect of the pulsing was mainly to reduce confusion effects, one might expect that performance would initially improve with increasing inter-pulse interval or rise/fall time, and then reach an asymptote when confusion was completely resolved. The data show no sign of an asymptote, except when performance was close to ceiling. For the $0.75-E$ spacing, performance was close to chance when the target component was not pulsed, as would be expected if the components were completely unresolved for this spacing. However, when the target was pulsed, the mean scores improved from about $67 \%$ to $80 \%$ when the inter-pulse interval was increased from 50 to $100 \mathrm{~ms}$.

The pattern of results is consistent with an explanation based on recovery from adaptation, as described in Sec. I. According to this explanation, turning the target tone off momentarily leads to a recovery from adaptation, so that the neural response to the target is increased relative to that for adjacent (non-pulsed) partials when the target is turned back on. The amount of recovery would increase as the on-off ramps were made longer and as the inter-pulse interval was increased. However, it is hard to rule out a contribution from resolution of confusion caused by an increase in the salience of the target component as the inter-pulse interval or rise/fall time were made longer. 


\section{EXPERIMENT 2: DETECTION OF WHICH COMPLEX TONE CONTAINS A PULSED PARTIAL}

According to the explanation of the results based on recovery from adaptation, as described above, the pulsing of the target is beneficial because it leads to an enhanced neural response to the target, such that the response of neurons tuned close to the frequency of the target becomes higher than the response of neurons tuned to the frequencies of adjacent partials. If this explanation is correct, then the pulsing should affect the ability to detect the pulsed target in the complex tone in a similar way that it affects the ability to "hear out" the target partial, as measured in experiment 1. Experiment 2 was conducted to test this prediction. The stimuli and task were designed so that there was little uncertainty about what was to be detected. In experiment 1 , there was a potential perceptual confusion because the frequency of the probe had to be compared with the frequency of the closest partial in the complex, and it may not have been obvious to the listener which one was the closest. In experiment 2 , the probe frequency coincided exactly with the frequency of one of the partials in the complex. The other source of confusion mentioned in Sec. I, namely, perceptual fusion of the partials, should have been small in both experiments, for the following reasons: (1) The stimuli were inharmonic, eliminating the tendency for perceptual fusion caused by harmonicity; (2) the stimuli were relatively long, whereas perceptual fusion caused by synchronous gating is strong mainly for short-duration signals (Moore et al., 1986). Thus the results of experiment 2 should have been minimally affected by confusion effects. We reasoned that, if the pattern of results was similar for experiments 1 and 2, this would support the idea that the effects of pulsing found in experiment 1 were mainly due to recovery from adaptation rather than to resolution of confusion.

\section{A. Stimuli, procedure, and subjects}

A two-interval two-alternative forced-choice procedure was used. In each interval a probe tone with 1000-ms overall duration (including 20-ms raised-cosine ramps) was followed by a complex tone. The complex tones were the same as those used in experiment 1 , and had the same overall duration of $1000 \mathrm{~ms}$. The frequency of the probe tone was equal to the frequency of one of the partials in the complex tone, the target. Thus, there was no uncertainty about which partial in the complex the probe should be compared to. In both intervals of a trial, the probe tone was pulsed on and off with one of the pulse patterns corresponding to conditions 2-6 of experiment 1 . In both intervals, all of the components in the complex tone except the target were uninterrupted over the 1000-ms duration. In one interval, selected randomly, the target tone was pulsed on and off with the same pattern as the probe. In the other interval, the target was uninterrupted over the 1000-ms duration. The task of the subject was to identify the interval in which the target was pulsed. The frequency of the probe tone and the target tone was randomly selected on each trial from 1 of the 11 possible frequencies, as indicated in Table I. In this experiment, the probe served to indicate the frequency region in which to listen for the pulsing and also the pulsing pattern to listen for. To make the task as similar as possible to that for experiment 1 , the frequencies of all partials in the complex tone were randomly varied (roved) from trial to trial by multiplying them by a factor randomly chosen within the range $0.9-1.1$, while keeping the frequency ratios between partials fixed. The frequency of the probe was multiplied by the same factor. The subjects were the same as for experiment 1 . Each partial in a complex was probed five times in a given run. Five runs were obtained for each complex tone, giving a total of 25 judgments for each partial.

\section{B. Results and discussion}

Again, the general pattern of results was similar across subjects. Mean results are shown in Fig. 3. The scores are plotted as a function of partial number, with pulse pattern as parameter. Each panel shows results for one spacing. Although the results were somewhat "noisy," the overall pattern was similar to that found in experiment 1 . This is illustrated in Fig. 4, which shows results averaged across all of the inner partials except the tenth and plotted as a function of spacing, with pulse pattern as a parameter. Scores improved with increasing spacing and performance was strongly affected by the pulse pattern, being worst for condition 2 (10-ms ramps, no gaps) and best for condition 6 (20-ms ramps and 100-ms gaps).

To assess the statistical significance of these effects, the data were subjected to a rationalized arcsine transform and then to a within-subjects ANOVA, with factors type of pulsing (five types), partial number (2-9), and spacing $(0.75,1$, or $\left.1.5 \mathrm{ERB}_{\mathrm{N}}\right)$. The main effect of type of pulsing was significant: $F(4,12)=47.38, p<0.001$. The main effect of partial number was significant: $F(7,21)=2.80, p=0.032$. The main effect of spacing was significant: $F(2,6)=21.78, p=0.002$. No interactions were significant.

\section{Comparison of results for experiments 1 and 2}

To compare the results for the two experiments, a within-subjects ANOVA was conducted on the RAUtransformed data with factors task ("hearing out" of partial as in experiment 1 , or "detection of pulsing" as in experiment 2 ), spacing, pulsing type, and partial number (2-9). The data for condition 1 from experiment 1 (where the probe and target were not pulsed) were excluded, as there was no corresponding condition in experiment 2.

The effect of spacing was significant, $F(2,6)=34.01, p$ $<0.001$, as were the effects of pulsing pattern, $F(4,12)$ $=90.16, p<0.001$, and partial number, $F(7,21)=3.04, p$ $=0.023$. The effect of task was not significant: $F(1,3)$ $=3.46, p=0.16$. In other words, a given partial could be heard out (experiment 1) with about the same accuracy as the interval containing the pulsed target could be identified in experiment 2. The grand mean scores in RAU were 82.4 for experiment 1 and 89.2 for experiment 2. However, it should be noted that two subjects did show somewhat better performance for experiment 2 than for experiment 1 . The other two subjects showed similar performance for the two experi- 


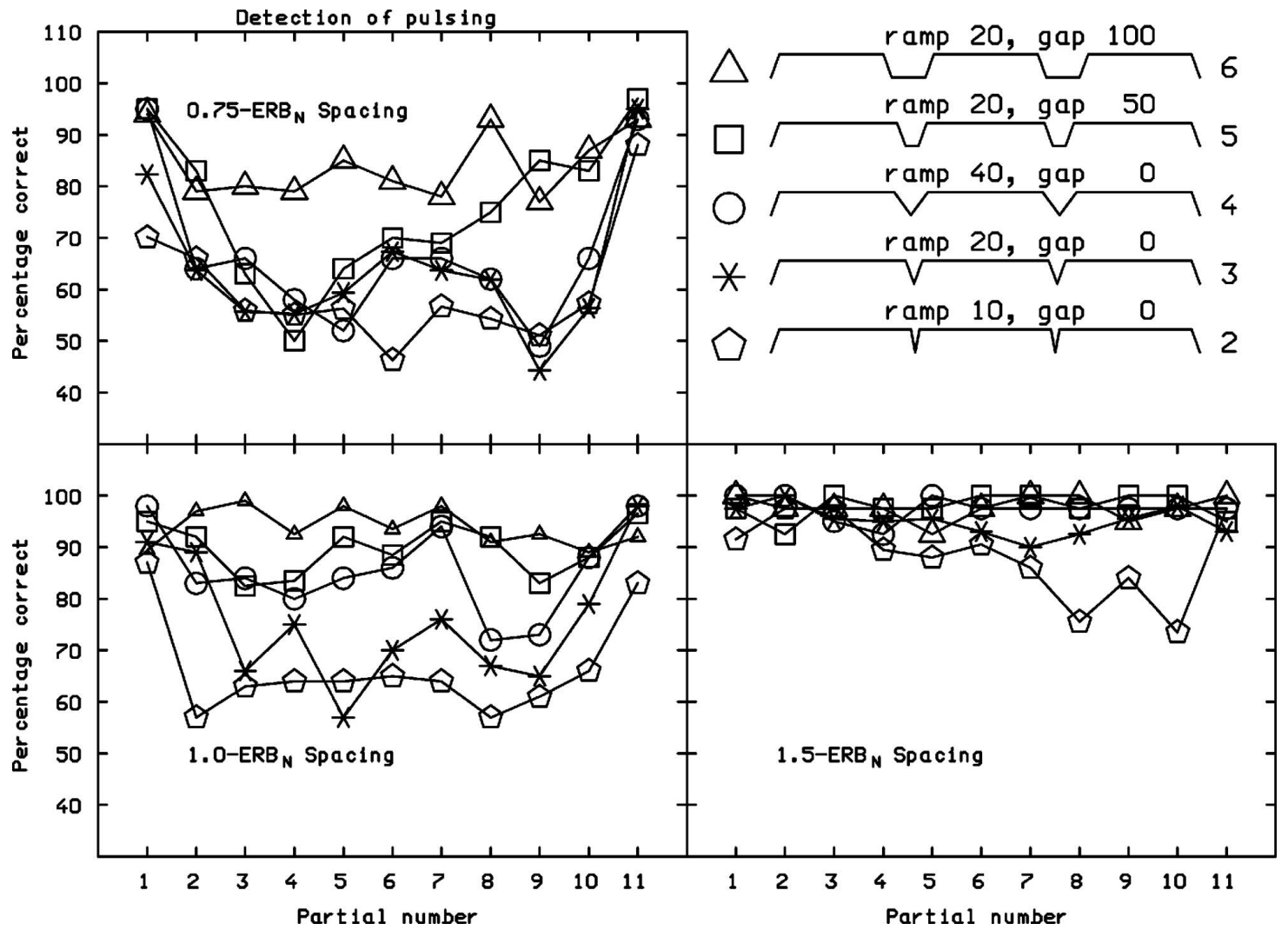

FIG. 3. As Fig. 1, but showing scores for experiment 2, in which subjects had to identify which of two complex tones contained a pulsed partial.

ments. There was a significant interaction between task and pulsing pattern; $F(4,12)=4.2, p=0.024$, although this accounted for less than $2 \%$ of the variance in the data.

\section{INTERPRETING THE RESULTS USING A MODEL OF AUDITORY PROCESSING}

The similar pattern of results for the two experiments could be explained in terms of the increasing perceptual salience of the gaps in the pulsed partial as the gaps were made longer. The increasing salience could draw attention to the pulsed partial, making it both easier to hear out and to detect. This explanation would be consistent with previous data on

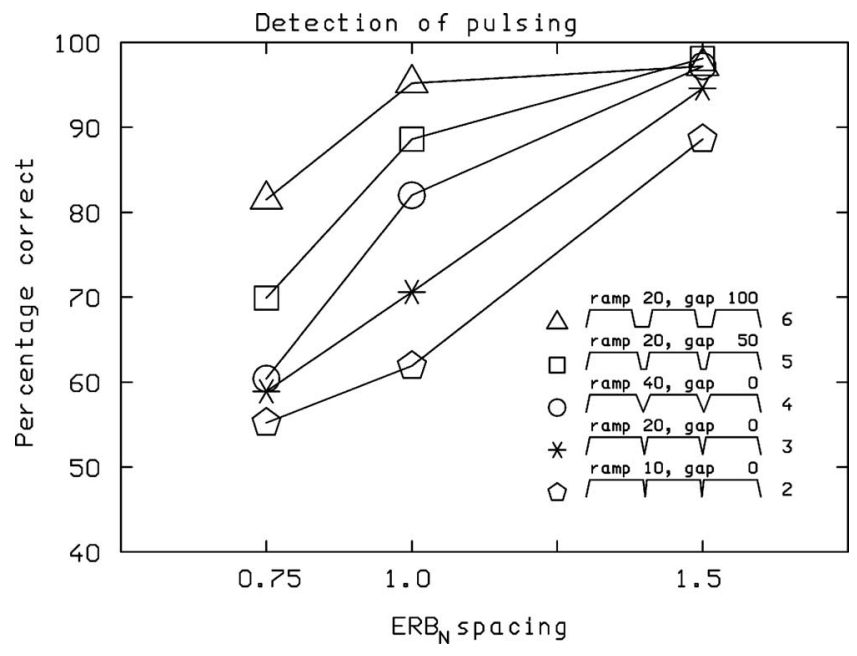

FIG. 4. As Fig. 2, but showing results for experiment 2. the detection of gaps or decrements in level of sounds, which have been modeled in terms of the detection of dips in the output of a sliding temporal integrator (Moore et al., 1988; Plack and Moore, 1991; Moore et al., 1993), without taking into account processes associated with adaptation or a release from adaptation. However, the stimuli in those experiments mostly involved gaps or decrements in narrowband sounds or sounds in which the gap or decrement occurred across the whole stimulus spectrum. For multiple-component sounds with closely spaced components in which only one component has gaps, as in the present experiments, the fact that the response to the pulsed component would momentarily exceed the response to adjacent components (as a result of release from adaptation), or exceed the on-going response within the channel tuned to the pulsed component, may be highly perceptually relevant.

In this section, we use a model of auditory processing to illustrate the effects that adaptation might have on the detection of the pulsed partial, as tested in experiment 2. We use the model described by Dau et al. (1996a), which has been shown to account for a broad range of spectral and temporal masking data (Dau et al., 1996b). This model includes a linear auditory filter bank and an adaptation stage, which is important for accounting for temporal masking effects (Dau et al., 1996b). The original version of the model has been modified to include a modulation filter bank (Dau et al., 1997a, 1997b) and a more complex basilar-membrane (BM) stage to simulate cochlear compression (Jepsen et al., 2008). However, we used the original version of the model (Dau et al., 1996a) for simplicity, since it was thought that BM 


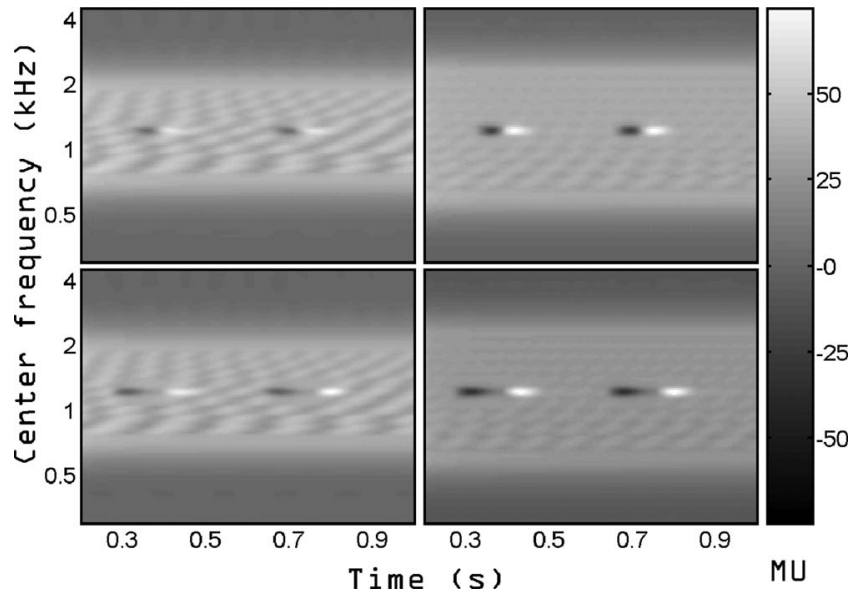

FIG. 5. IR for the complex tones used in four different conditions, with the sixth partial pulsed. Time is plotted on the $x$-axis, channel CF is plotted on the $y$-axis, and darkness-brightness shows excitation in MUs (see scale on the right). The spacing of the partials was either $0.75 E$ (left) or $1 E$ (right). The two pulsing conditions were 40-ms ramps, 0-ms gaps (top) or 20-ms ramps, 100-ms gaps (bottom).

compression and processing in the modulation filter bank would not have a major influence on the detection of a pulsed partial.

The model consists of the following.

(1) An array of linear gammatone filters (Patterson et al., 1995), to simulate BM processing. The bandwidths of the filters had the values suggested by Glasberg and Moore (1990).

(2) Half-wave rectification followed by a lowpass filter with a cut-off frequency at $1 \mathrm{kHz}$, to simulate the action of the inner hair cells.

(3) A non-linear adaptation stage that consists of five feedback loops with time constants ranging from 5 to 500 ms.

(4) A lowpass filter with a cutoff frequency at $8 \mathrm{~Hz}$. This acts to emphasize energy from low-frequency modulation in the envelope of the signal.

For full details of each stage, the reader is referred to Dau et al. (1996a). It should be noted that the model as used here does not take confusion effects into account.

We focus on illustrating the effects of adaptation within the model, by using displays of its internal representation (IR). Hence we disregard the decision mechanism of the original model. The IR display is a spectrogram-like plot of the internal signal excitation in model units (MUs) as a function of filter center frequency (CF) and time. Figure 5 shows IRs for the complex tones used in four different conditions, with the sixth partial pulsed. The contrast of the display has been chosen such that a change in level of about $1 \mathrm{~dB}$ would just be visible. The initial part of the IR, from 0 to $0.2 \mathrm{~s}$, is not shown. The spacing of the partials was either $0.75 E$ (left) or $1 E$ (right). The two pulsing conditions were $40-\mathrm{ms}$ ramps, 0-ms gaps (condition 4, top) or 20-ms ramps, 100-ms gaps (condition 6, bottom). In each case, the channel tuned to the frequency of the pulsed partial shows a decrease in excitation when the partial is turned off, and then a distinct increase in excitation, above the amount of excitation during the steady

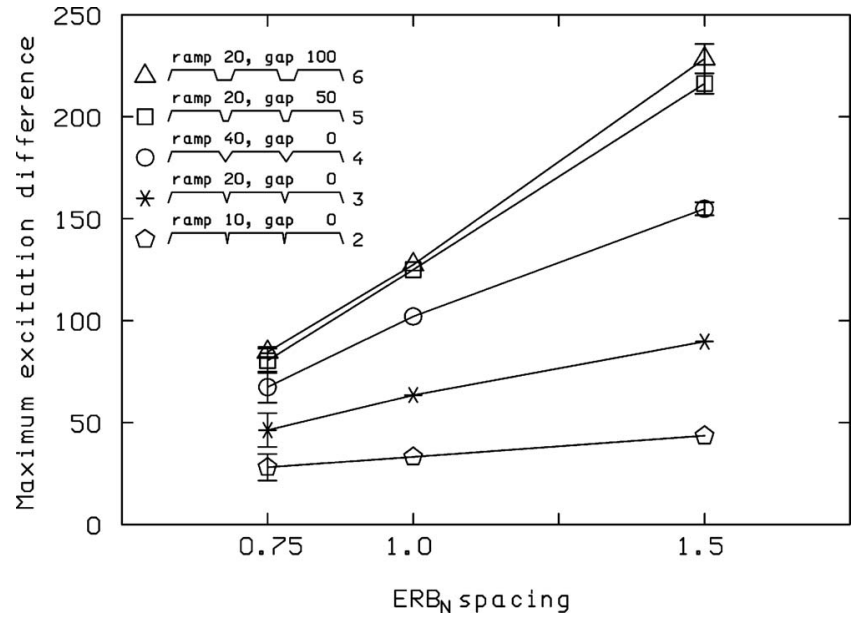

FIG. 6. The MED plotted as a function of $\mathrm{ERB}_{\mathrm{N}}$-spacing, with pulsing condition as parameter. The data points are based on the mean values of the MED obtained when partial numbers 3, 6, and 9 were pulsed. Error bars show the standard deviation of the MED across the three partials.

parts of the sound, just after the partial is turned back on. This increase corresponds to a release from adaptation. The release from adaptation is greater for the wider $\mathrm{ERB}_{\mathrm{N}}$ spacing (compare the left and right panels) and is greater for the longer gap duration (compare the top and the bottom panels).

For the $0.75 E$ and $1 E$ spacings, the IR showed only small across-CF variation when no partial was pulsed; the "ripple" in the IR, which provides an indication of the degree to which the partials were resolved, was about 1.5 MU for the $0.75 E$ spacing and $1.9 \mathrm{MU}$ for the $1 E$ spacing, while the mean excitation was about $43 \mathrm{MU}$ (the ripple was larger, at about $6 \mathrm{MU}$ for the $1.5 E$ spacing). These small variations are consistent with the idea that, for the spacings of $0.75 E$ and $1 E$, the partials were barely, if at all, resolved. Pulsing a partial off and on produced more substantial decreases and increases in excitation in the channel tuned to the frequency of the pulsed partial; the increases above the amount of excitation during the steady part of the sound are the result of a release from adaptation. For example, for the condition with 20 -ms ramps and 100-ms gaps, the increases in excitation produced by the pulsing relative to the excitation during the steady parts of the stimulus (averaged across channels tuned to the third, sixth, and ninth partials) were $28 \mathrm{MU}$ for the $0.75 E$ spacing, $57 \mathrm{MU}$ for the $1 E$ spacing, and $107 \mathrm{MU}$ for the $1.5 E$ spacing.

To quantify the effect of pulsing of the partial on the IR, we considered only the output of the channel tuned to the frequency of the pulsed partial. This seems reasonable, since in experiment 2 the pulsed partial was presented in isolation before the complex tone, and this would have directed attention to the relevant channel. The first $200 \mathrm{~ms}$ of the IR was disregarded, as this was strongly affected by the onset response to all partials. We used as a measure the maximum excitation difference (MED), defined as the difference in excitation in MU between the minimum excitation in the channel (which occurred just after the partial was turned off) and the peak excitation in the channel (which occurred just after the partial was turned back on). Figure 6 shows the MED plotted as a function of $\mathrm{ERB}_{\mathrm{N}}$-spacing, with pulsing condi- 


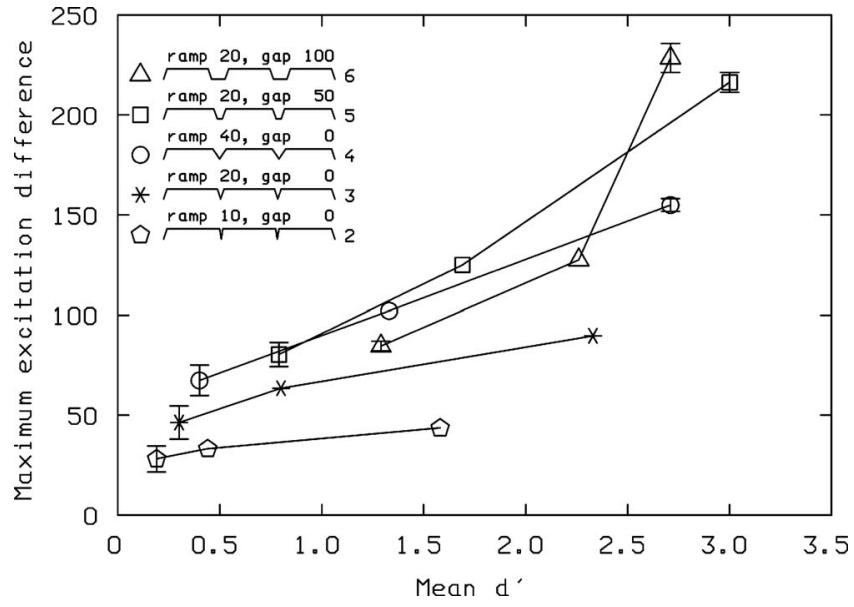

FIG. 7. The MED plotted against the corresponding mean $d^{\prime}$ value obtained for each pulsing condition and spacing in experiment 2. Error bars show the standard deviation of the MED across partials 3, 6, and 9.

tion as parameter. The data points are based on the mean values of the MED obtained when partial numbers 3, 6, and 9 were pulsed. Error bars show \pm 1 standard deviation. For each pulsing condition, the MED increases as the spacing of the partials increases. More importantly, the MED increases as the ramp duration and/or the gap duration increases. The ordering of the functions for the different pulsing conditions is the same as for the experimental data plotted in Fig. 4.

Figure 7 shows the MED plotted against the corresponding mean $d^{\prime}$ value obtained for each pulsing condition and spacing in experiment 2 . If performance were perfectly accounted for by the MED, then the functions for the different pulsing conditions would all lie on top of one another. Clearly, this is not the case. In particular, the MEDs for the pulsing conditions with 0 -ms gaps and ramps of 10 or $20 \mathrm{~ms}$ fall below those for the other pulsing conditions. Hence, the model predicts that the salience of the pulsing in these two conditions would be smaller relative to the other conditions than is actually the case. This may reflect a limitation in the accuracy of the model in simulating adaptation effects. It may also reflect some other aspects of auditory processing that are not simulated by the model, such as suppression. Nevertheless, the modeling clearly illustrates how pulsing a partial on and off can provide cues for identifying the channel corresponding to the pulsed partial, even when the partial is not resolved by the auditory filters.

\section{DISCUSSION}

We argued in Sec. I that if resolution of confusion were the sole effect of pulsing the target in experiment 1 , then one would expect performance to reach a plateau if the interpulse interval was sufficiently long. For closely spaced partials, for which peripheral resolvability should limit performance, this plateau should occur for a performance level well below $100 \%$. In fact, in experiment 1, performance increased progressively with increasing inter-pulse interval, and there was no sign of a plateau, even for the closely spaced components. However, a similar pattern of performance was observed in experiment 2, indicating that the ability to detect the pulsing also increased progressively with increasing inter-pulse interval. Since the pulsing was never perfectly detectable at the smallest spacing of the partials, the cue provided by the pulsing in experiment 1 would not have been fully effective in resolving confusion. This makes it difficult to draw any strong conclusions from the lack of a plateau in the data for the smallest spacing.

Experiment 2 was designed so that there was little uncertainty about what was to be detected; the probe tone indicated the frequency and pulsing pattern of the target tone in the complex. Also, the partials in the complex were not harmonically related, so perceptual fusion based on harmonicity could not have played a role. Thus, the results should have been minimally affected by attentional or cognitive factors. The pattern of results for experiment 2 was very similar to that for experiment 1 , and, excluding the special case of the tenth (second highest) partial, overall performance was similar for the two experiments. This suggests that the change in performance with pulsing pattern in experiment 1 was not a consequence of the pulse patterns varying in the extent to which they reduced uncertainty about what to listen for. Rather, the results are consistent with the idea that, in both experiment 1 and experiment 2, increasing the rise/fall time or inter-pulse interval of the target tone produced a greater recovery from adaptation, and that this led to improved performance. The results of the modeling described in Sec. IV support this interpretation.

If this interpretation is correct, it means that measurement of the ability to hear out a partial in a complex tone when the target partial is pulsed on and off does not give a valid indication of how well that partial can be heard out in the more common situation when the target tone is not pulsed. Rather, the method using a pulsing target leads to an over-estimate of the ability to hear out a partial. The present results are consistent with earlier results (Moore and Ohgushi, 1993; Moore et al., 2006c) indicating that, in the absence of pulsing, a target partial needs to be separated from neighboring partials by between $1 E$ and $1.5 E$ to be heard out with $75 \%$ accuracy, although a somewhat greater separation is needed as the frequency of the target partial increases above about $1500 \mathrm{~Hz}$, probably because the contribution of phase locking information decreases at high frequencies (Hartmann et al., 1990; Hartmann and Doty, 1996; Moore et al., 2006c). For harmonic complex tones with equal-amplitude (non-pulsed) harmonics, the results imply that only the lowest 5-8 harmonics can be heard out for complex tones with fundamental frequencies in the range 100-400 Hz, as concluded earlier by Plomp (1964) and Plomp and Mimpen (1968). For example, for a fundamental frequency of $100 \mathrm{~Hz}$, the seventh and eighth harmonics are separated by slightly less than $1 E$, and so would be barely, if at all, resolved.

This conclusion has important implications for theories of pitch perception. It has been shown in several studies that the ability to discriminate the fundamental frequency of harmonic complex tones is worse when the tones contain only high harmonics than when they contain low harmonics (Hoekstra and Ritsma, 1977; Hoekstra, 1979; Moore and Glasberg, 1988; Houtsma and Smurzynski, 1990; Carlyon and Shackleton, 1994; Shackleton and Carlyon, 1994). The 
transition from good to poor performance as the rank, $N$, of the lowest harmonic increases, occurs roughly over the range $N=8-14$ (Hoekstra and Ritsma, 1977; Houtsma and Smurzynski, 1990; Bernstein and Oxenham, 2003, 2008; Moore et al., 2006b). One interpretation of the "transition region" is that it reflects a progressive loss of sensitivity to the temporal fine structure of the stimuli (Moore and Moore, 2003b; Moore et al., 2006a; 2006b; Hopkins and Moore, 2007; Ives and Patterson, 2008). An alternative interpretation is that it reflects a transition from resolved to unresolved harmonics (Hoekstra and Ritsma, 1977; Hoekstra, 1979; Carlyon and Shackleton, 1994), or from harmonics that would usually be resolved to harmonics that would usually be unresolved (Bernstein and Oxenham, 2003, 2008). The present results support the former interpretation, since it appears that harmonics above the eighth are not usually resolved.

These results also have implications for the interpretation of a test of sensitivity to temporal fine structure described by Hopkins and Moore (2007) and Moore and Sek (2009). The validity of this test depends on the partials in the stimuli being unresolved. The task involves discriminating a harmonic complex tone $(\mathrm{H})$, with fundamental frequency $\mathrm{F} 0$, from a similar tone in which all components are shifted up in frequency by the same amount in hertz, $\Delta F$, so as to create an inharmonic tone (I). The shift does not change the envelope repetition rate, but it results in a change in the temporal fine structure of the sound. As argued above, for tones with equal-amplitude harmonics, harmonics with numbers above 8 are not resolved. However, a harmonic higher than the eighth may be resolved when it has a higher amplitude than adjacent harmonics or when it is the highest or lowest harmonic in the complex tone. To generate complex tones that contained only unresolved harmonics, Hopkins and Moore (2007) and Moore and Sek (2009) passed the complex tones through a fixed bandpass filter with moderately steep slopes of $30 \mathrm{~dB} /$ octave. A similar strategy has been used by previous researchers (Hoekstra, 1979; Carlyon and Shackleton, 1994; Shackleton and Carlyon, 1994; Moore and Moore, 2003a). To prevent detection of harmonics well down on the skirts of the resulting spectrum, and to prevent the detection of combination tones, the stimuli were presented in a background noise.

An important question is: where does the bandpass filter need to be positioned to ensure that no harmonics are resolved? In one of the conditions of Hopkins and Moore (2007), the filter was centered on the 11th harmonic, and the lowest harmonic within the passband was the 9th. It is likely that one or two harmonics falling on the lower skirt of the filter would have been audible in the presence of the background noise. Thus the lowest audible harmonic would have been the seventh or the eighth. These harmonics would have been barely, if at all, resolved, but it is just possible that they provided usable spectral cues. However, our more recent work (Moore et al., 2009) has shown that it is possible to perform the test (albeit with poorer performance), when the bandpass filter is centered on the 13th or 15th harmonic. In the latter case, the lowest audible harmonic would have been the 11th, and this would certainly have been unresolved.
Thus, performance of the test does not seem to depend on information from resolved harmonics when the CF of the bandpass filter is sufficiently high.

\section{CONCLUSIONS}

The ability to hear out a partial in a complex tone was strongly affected by pulsing the target partial on and off, and by varying the ramp times and off times of the pulses; longer ramp times and longer inter-pulse intervals led to improved performance. The pattern of results was similar to that obtained when the task was to detect which of two complex tones contained a pulsed partial. The results are more consistent with an explanation for the effect of the pulsing in terms of recovery from adaptation than with an explanation in terms of attentional or cognitive factors. Results obtained using a model of auditory processing are consistent with this interpretation. Overall, the results suggest that measurement of the ability to hear out a partial in a complex tone when the target partial is pulsed on and off does not give a valid indication of how well that partial can be heard out in the more common situation when the target tone is not pulsed.

\section{ACKNOWLEDGMENTS}

This work was supported by the MRC (U.K.). We thank Hedwig Gockel, Kathryn Hopkins, Richard Freyman, Andrew Oxenham, and an anonymous reviewer for helpful comments on an earlier version of this paper.

Bernstein, J. G., and Oxenham, A. J. (2003). "Pitch discrimination of diotic and dichotic tone complexes: Harmonic resolvability or harmonic number?" J. Acoust. Soc. Am. 113, 3323-3334.

Bernstein, J. G., and Oxenham, A. J. (2006). "The relationship between frequency selectivity and pitch discrimination: Effects of stimulus level," J. Acoust. Soc. Am. 120, 3916-3928.

Bernstein, J. G., and Oxenham, A. J. (2008). "Harmonic segregation through mistuning can improve fundamental frequency discrimination," J. Acoust. Soc. Am. 124, 1653-1667.

Carlyon, R. P., and Shackleton, T. M. (1994). "Comparing the fundamental frequencies of resolved and unresolved harmonics: Evidence for two pitch mechanisms?" J. Acoust. Soc. Am. 95, 3541-3554.

Dau, T., Kollmeier, B., and Kohlrausch, A. (1997a). "Modeling auditory processing of amplitude modulation. I. Detection and masking with narrowband carriers," J. Acoust. Soc. Am. 102, 2892-2905.

Dau, T., Kollmeier, B., and Kohlrausch, A. (1997b). "Modeling auditory processing of amplitude modulation. II. Spectral and temporal integration," J. Acoust. Soc. Am. 102, 2906-2919.

Dau, T., Püschel, D., and Kohlrausch, A. (1996a). "A quantitative model of the 'effective' signal processing in the auditory system. I. Model structure," J. Acoust. Soc. Am. 99, 3615-3622.

Dau, T., Püschel, D., and Kohlrausch, A. (1996b). "A quantitative model of the 'effective' signal processing in the auditory system. II. Simulations and measurements," J. Acoust. Soc. Am. 99, 3623-3631.

Fine, P. A., and Moore, B. C. J. (1993). "Frequency analysis and musical ability," Music Percept. 11, 39-53.

Glasberg, B. R., and Moore, B. C. J. (1990). "Derivation of auditory filter shapes from notched-noise data," Hear. Res. 47, 103-138.

Gockel, H., Moore, B. C. J., Carlyon, R. P., and Plack, C. J. (2007). "Effect of duration on the frequency discrimination of individual partials in a complex tone and on the discrimination of fundamental frequency," $\mathrm{J}$. Acoust. Soc. Am. 121, 373-382.

Hartmann, W. M., and Doty, S. L. (1996). "On the pitches of the components of a complex tone," J. Acoust. Soc. Am. 99, 567-578.

Hartmann, W. M., and Goupell, M. J. (2006). "Enhancing and unmasking the harmonics of a complex tone," J. Acoust. Soc. Am. 120, 2142-2157. Hartmann, W. M., McAdams, S., and Smith, B. K. (1990). "Hearing a mistuned harmonic in an otherwise periodic complex tone," J. Acoust. Soc. 
Am. 88, 1712-1724.

Helmholtz, H. L. F. (1954). On the Sensations of Tone (Dover, New York).

Hoekstra, A. (1979). "Frequency discrimination and frequency analysis in hearing," Ph.D. thesis, Institute of Audiology, University Hospital, Groningen, The Netherlands.

Hoekstra, A., and Ritsma, R. J. (1977). "Perceptive hearing loss and frequency selectivity," in Psychophysics and Physiology of Hearing, edited by E. F. Evans and J. P. Wilson (Academic, London, England).

Hopkins, K., and Moore, B. C. J. (2007). "Moderate cochlear hearing loss leads to a reduced ability to use temporal fine structure information," $\mathrm{J}$ Acoust. Soc. Am. 122, 1055-1068.

Houtsma, A. J. M., and Smurzynski, J. (1990). "Pitch identification and discrimination for complex tones with many harmonics," J. Acoust. Soc. Am. 87, 304-310.

ISO 389-8 (2004). Acoustics-Reference zero for the calibration of audiometric equipment-Part 8: Reference equivalent threshold sound pressure levels for pure tones and circumaural earphones (International Organization for Standardization, Geneva).

Ives, D. T., and Patterson, R. D. (2008). "Pitch strength decreases as F0 and harmonic resolution increase in complex tones composed exclusively of high harmonics," J. Acoust. Soc. Am. 123, 2670-2679.

Jepsen, M. L., Ewert, S. D., and Dau, T. (2008). "A computational model of human auditory signal processing and perception," J. Acoust. Soc. Am. 124, 422-438.

Johnson, D. H. (1980). "The relationship between spike rate and synchrony in responses of auditory-nerve fibers to single tones," J. Acoust. Soc. Am. 68, 1115-1122.

Moore, B. C. J. (1973). "Some experiments relating to the perception of complex tones," Q. J. Exp. Psychol. 25, 451-475.

Moore, B. C. J. (2003). An Introduction to the Psychology of Hearing, 5th ed. (Academic, San Diego).

Moore, B. C. J., Alcántara, J. I., and Dau, T. (1998). "Masking patterns for sinusoidal and narrowband noise maskers," J. Acoust. Soc. Am. 104, 1023-1038.

Moore, B. C. J., and Glasberg, B. R. (1988). "Effects of the relative phase of the components on the pitch discrimination of complex tones by subjects with unilateral cochlear impairments," in Basic Issues in Hearing, edited by H. Duifhuis, H. Wit, and J. Horst (Academic, London).

Moore, B. C. J., Glasberg, B. R., Flanagan, H. J., and Adams, J. (2006b) "Frequency discrimination of complex tones; assessing the role of component resolvability and temporal fine structure," J. Acoust. Soc. Am. 119, 480-490.

Moore, B. C. J., Glasberg, B. R., and Hopkins, K. (2006a). "Frequency discrimination of complex tones by hearing-impaired subjects: Evidence for loss of ability to use temporal fine structure information," Hear. Res. 222, 16-27.

Moore, B. C. J., Glasberg, B. R., Low, K.-E., Cope, T., and Cope, W (2006c). "Effects of level and frequency on the audibility of partials in inharmonic complex tones," J. Acoust. Soc. Am. 120, 934-944.

Moore, B. C. J., Glasberg, B. R., and Peters, R. W. (1986). "Thresholds for hearing mistuned partials as separate tones in harmonic complexes," J. Acoust. Soc. Am. 80, 479-483.

Moore, B. C. J., Glasberg, B. R., Plack, C. J., and Biswas, A. K. (1988).
"The shape of the ear's temporal window," J. Acoust. Soc. Am. 83, 11021116

Moore, B. C. J., Glasberg, B. R., and Shailer, M. J. (1984). "Frequency and intensity difference limens for harmonics within complex tones," J. Acoust. Soc. Am. 75, 550-561.

Moore, B. C. J., and Moore, G. A. (2003a). "Discrimination of the fundamental frequency of complex tones with fixed and shifting spectral envelopes by normally hearing and hearing-impaired subjects," Hear. Res. 182, $153-163$.

Moore, B. C. J., and Ohgushi, K. (1993). "Audibility of partials in inharmonic complex tones," J. Acoust. Soc. Am. 93, 452-461.

Moore, B. C. J., Hopkins, K., and Cuthbertson, S. J. (2009). "Discrimination of complex tones with unresolved components using temporal fine structure information," J. Acoust. Soc. Am. (in press).

Moore, B. C. J., Peters, R. W., and Glasberg, B. R. (1993). "Detection of temporal gaps in sinusoids: Effects of frequency and level," J. Acoust. Soc. Am. 93, 1563-1570.

Moore, B. C. J., and Sek, A. (2009). "Development of a fast method for determining sensitivity to temporal fine structure," Int. J. Audiol. (in press).

Moore, G. A., and Moore, B. C. J. (2003b). "Perception of the low pitch of frequency-shifted complexes,” J. Acoust. Soc. Am. 113, 977-985.

Palmer, A. R., and Russell, I. J. (1986). "Phase-locking in the cochlear nerve of the guinea-pig and its relation to the receptor potential of inner haircells," Hear. Res. 24, 1-15.

Palmer, A. R., Summerfield, Q., and Fantini, D. A. (1995). "Responses of auditory-nerve fibers to stimuli producing psychophysical enhancement," J. Acoust. Soc. Am. 97, 1786-1799.

Patterson, R. D., Allerhand, M. H., and Giguère, C. (1995). "Time-domain modeling of peripheral auditory processing: A modular architecture and a software platform," J. Acoust. Soc. Am. 98, 1890-1894.

Plack, C. J., and Moore, B. C. J. (1991). "Decrement detection in normal and impaired ears," J. Acoust. Soc. Am. 90, 3069-3076.

Plomp, R. (1964). "The ear as a frequency analyzer," J. Acoust. Soc. Am. 36, $1628-1636$.

Plomp, R., and Mimpen, A. M. (1968). "The ear as a frequency analyzer II," J. Acoust. Soc. Am. 43, 764-767.

Roberts, B., and Bregman, A. S. (1991). "Effects of the pattern of spectral spacing on the perceptual fusion of harmonics," J. Acoust. Soc. Am. 90, 3050-3060.

Shackleton, T. M., and Carlyon, R. P. (1994). "The role of resolved and unresolved harmonics in pitch perception and frequency modulation discrimination," J. Acoust. Soc. Am. 95, 3529-3540.

Smith, R. L. (1977). "Short-term adaptation in single auditory-nerve fibers: Some poststimulatory effects," J. Neurophysiol. 49, 1098-1112.

Smith, R. L. (1979). "Adaptation, saturation and physiological masking in single auditory-nerve fibers," J. Acoust. Soc. Am. 65, 166-178.

Soderquist, D. R. (1970). "Frequency analysis and the critical band," Psychonomic Sci. 21, 117-119.

Studebaker, G. (1985). "A 'rationalized' arcsine transform,” J. Speech Hear. Res. 28, 455-462.

Westerman, L. A., and Smith, R. L. (1984). "Rapid and short-term adaptation in auditory nerve responses," Hear. Res. 15, 249-260. 